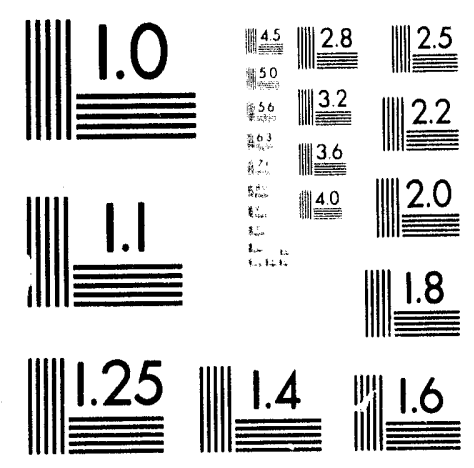



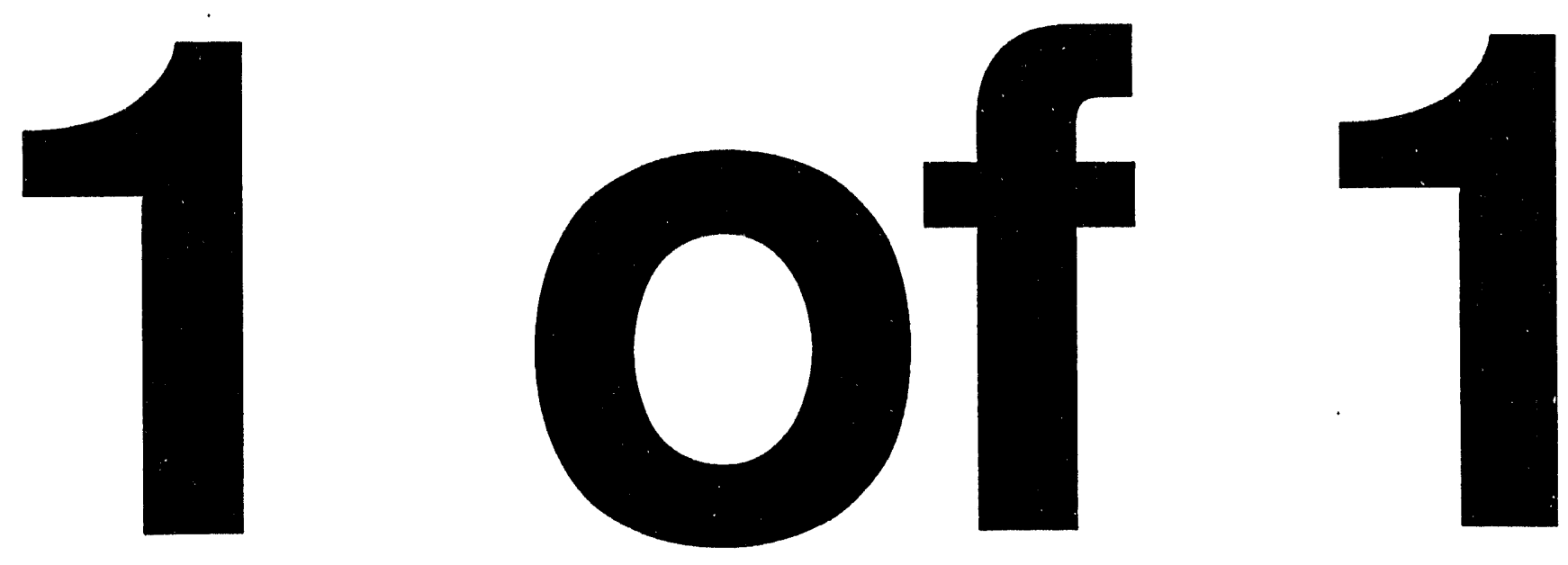


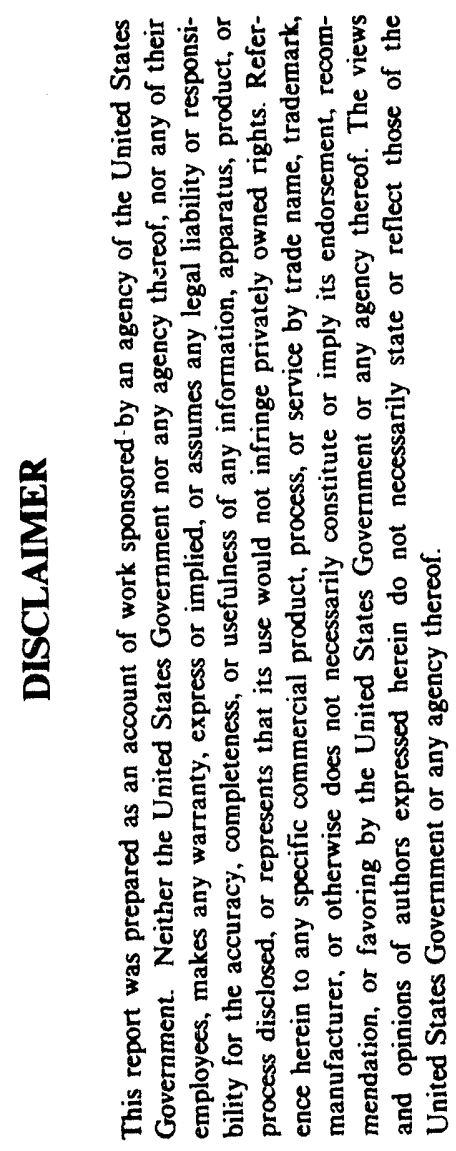

Submitted to:

1993 IEEE International Electron Devices Meeting

Washington, DC

December 5-8, 1993

\section{RF SYSTEM CONSIDERATIONS FOR ACCELERATOR PRODUCTION OF TRITIUM AND THE TRANSMUTATION OF NUCLEAR WASTE}

Author(s):

Paul J. Tallerico, Michael T. Lynch

\section{Los Alamos}

NATIONAL LABORATORY

\section{MASTER}

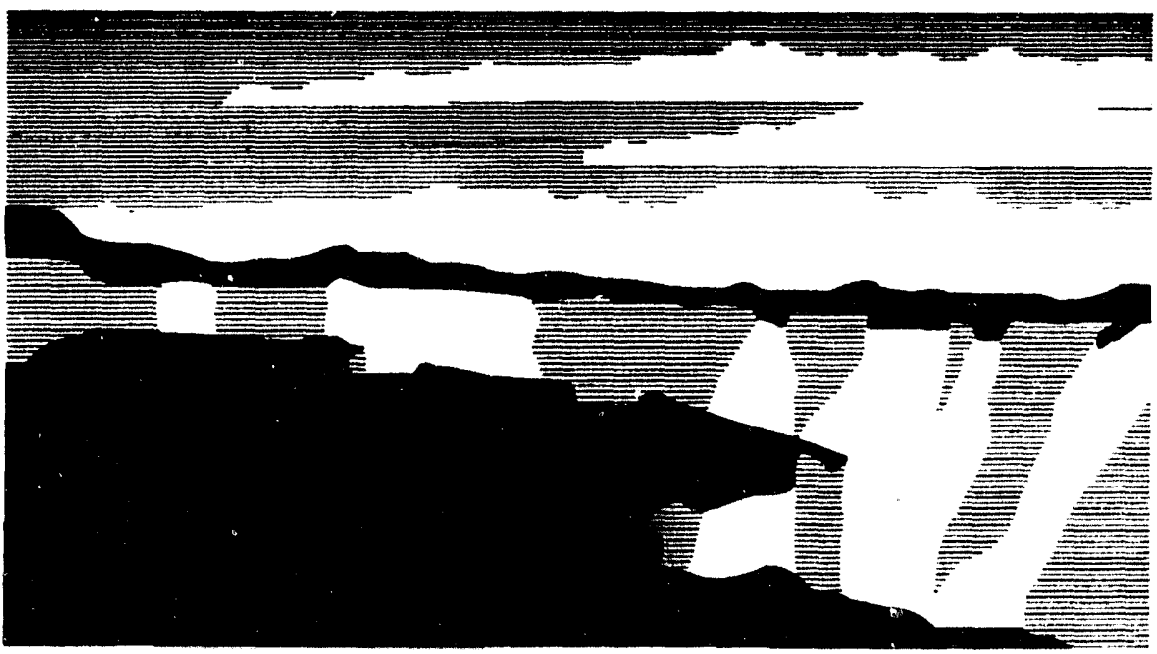

Los Alamos National Laboratory, an affirmative action/equal opportunity employer, is operated by the University of California for the U.S. Department of Energy under contract W.7405-ENG-36. By acceptance of this article, the publisher recognizes that the U.S. Government retains a nonexclusive, royalty-free license to publish or reproduce the published form of this contribution, or to allow others to do so, for U.S. Government purposes. The Los Alamos National Laboratory requests that the publisher identify this article as work performed under the auspices of the U.S. Department of Energy. 


\section{RF System Considerations for Accelerator Production of Tritium and the Transmutation of Nuclear Waste* \\ Paul J. Tallerico and Michael T. Lynch \\ Los Alamos National Laboratory, M/S H-827 \\ P.O. Box 1663, Los Alamos, NM 87545 (505) 667-1197}

\begin{abstract}
RF driven proton accelerators for the transmutation of nuclear waste (ATW) or for the production of tritium (APT) require unprecedented amounts of CW RF power at UHF frequencies(1). For both systems, the baseline design is for $246 \mathrm{MW}$ at $700 \mathrm{MHz}$ and $8.5 \mathrm{MW}$ at $350 \mathrm{MHz}$. The main technical challenges are how to design and build such a large system so that it has excellent reliability, high efficiency, and reasonable capital cost. The issues associated with the selection of the RF amplifier and the sizes of the power suppiits are emphasized in this paper.
\end{abstract}

\section{Introduction}

For the accelerator production of tritium and the accelerator transmutation of nuclear waste projects, a $200-\mathrm{MW}$ $\mathrm{CW}$ proton beam is used to produce neutrons at a target, and the neutrons then transmute material in desired ways. In the ATW project, a range of proton beams from 50 to $300 \mathrm{MW}$ has been considered, but the $200 \mathrm{MW}$ case is used here for illustrative purposes. The power in the proton beam in the design discussed here comes from the power grid, via dc power converters and a klystron RF system. The 254 MW RF systcm is almost larger by a factor of 10 than any yet built in terms of average power. The largest installations of CW RF power are in large electron-positron collider rings, the TRISTAN main ring at KEK in Japan and LEP at CERN in Geneva (2,3). TRISTAN currently has $30 \mathrm{MW}$ installed at $508 \mathrm{MHz}$, and LEP plans to increase its RF capability at $352.6 \mathrm{MHz}$ from $16 \mathrm{MW}$ to 32 MW by 1995 . Both of these rings use klystrons with from 1.0 to $1.3 \mathrm{MW} \mathrm{CW}$ output power.

Protons are almost 2000 times heavier than electrons, and thus low frequencies (below $1 \mathrm{GHz}$ ) are optimum for acceleration purposes. However, as the frequency decreases, - Work supported by the US Department of Energy with Los Alamos National Laboratory Program Development Funds. the accelerator becomes larger and more expensive, because the accelerator, like a microwave tube, scales with wavelength. Each accelerating cavity is $\beta \lambda / 2$ long, where $\beta$ is the proton velocity normalized to the speed of light and $\lambda$ is the RF wavelength. If the frequency is made too high, the cavities become too difficult to cool, and one must miniaturize the radial-focusing system, both of which increase the costs of the system. In practice, a broad frequency spectrum from about 200 to $400 \mathrm{MHz}$ is considered optimum for the low energy (and hence low- $\beta$ ) section of the accelerator. In the design presented here, the low energy end of the accelerator operates at $350 \mathrm{MHz}$, which is near the highest end of the allowable range yet at a frequency where reliable 1-MW CW klystrons have been developed.

An outline drawing of the accelerator along with its physical scale is shown in Fig. 1. Two proton injectors are used, and each provides $100 \mathrm{~mA} \mathrm{CW}$, at $75 \mathrm{keV}$.

The beams are then accelerated at $350 \mathrm{MHz}$ by a radio-frequency quadrupole (RFQ) accelerator and a drift tube linac (DTL) to $20 \mathrm{MeV}$. The two legs of the accelerator, which are out of phase by $180^{\circ}$, are combined in a funnel deflection system. The $200-m A$ combined beam is then accelerated by $700-\mathrm{MHz}$ RF power in a bridge-coupled drift tube linac (BCDTL) and then by a coupled-cavity linac $(\mathrm{CCL})$ to the final beam energy of $1000 \mathrm{MeV}$.

The details of the various accelerating structures are not of concern, but in the present design, they are normal-conducting, standing-wave structures. This latter property, which provides one technical challenge, means that each accelerator section behaves like a parallel LRC circuit: when it is empty, it reflects microwave power for time durations that are small compared to $1 / Q$.

The coupling between the accelerator tanks and the klystrons is made with an iris, which is designed so that there is a good match at the design beam curreni. When 


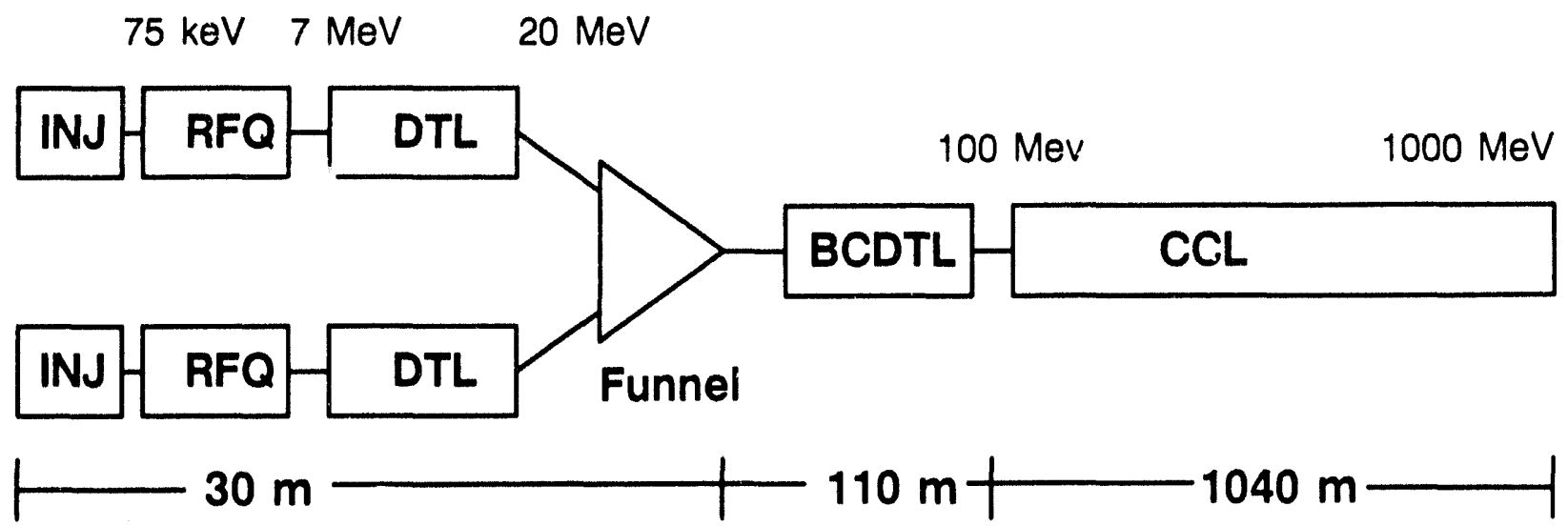

Fig. 1. Overall schematic of the APT/ATW accelerator.

the beam is accelerated, the proton beam is (to first order) a resistor in parallel with the first resistor, which is the measure of the Ohmic losses in the cavity.

A significant technical challenge is to maintain the cavity fields to a tolerance of less than $\pm 1 \%$ in amplitude and less than $1^{\circ}$ in phase, with a bandwidth above $100 \mathrm{kHz}$. This control problem is solved with a pick-up loop in each cavity and a fast analog-feedback loop around each klystron. The necessity for accurate control of the fields forces the operating point of the klystrons to be less than the saturated output power, and a control margin of $10 \%$ is required, which significantly reduces the overall efficiency of the system. Another problem with proton accelerators is that the proton's $\beta$ keeps changing with energy until the proton energy is well above the rest mass energy of $938 \mathrm{MeV}$. To overcome this, the entire accelerator structure has been designed to be synchronous with the local value of $\beta$. However, if a single RF amplifier fails, the synchronous property of the accelerator is lost for all higher energies. This makes the proton accelerator much less tolerant of a failed amplifier than an electron machine, and it is correspondingly more difficult to meet a given reliability goal.

\section{Baseline Technical Choices}

The major problems encountered in designing the RF system are the choice of RF amplifier, the size of the RF amplifier, the number of dc power supplies, optimization of dc-to-RF efficiency, and how to design the system for high availability and moderate cost. In the design work to date, these problems appear solvable.
The size of the amplifier was constrained by the fact that the accelerator designers would like to have hundreds of phase and amplitude controls along the machine to accurately control the proton beam position in phase space. Because the machine requires a minimum of $254 \mathrm{MW}$ of $\mathrm{RF}$ power, plus a $10 \%$ allowance for control margin, plus a $5 \%$ margin for power supply ripple and waveguide system losses, a 1 to $1.25 \mathrm{MW}$ saturated power for the amplifier was chosen. The list of amplifiers as used on the accelerator is shown in Table 1. The total number of amplifiers is 376 , and the amplifier of choice is the klystron, since it is the only amplifier that is a viable, demonstrated performer at these frequencies and power levels. Although the klystron represents rather old technology, the existing 1-MW klystrons at CERN and at TRISTAN are demonstrating saturated efficiencies of 65 to $68 \%$, along with mean time between failures (MTBFs) of at least 20,000 hours. While a larger amplifier would probably reduce system costs, a higher-powered klystron would operate at higher voltages, and have more power density in the beam and through the output window.

The alternative method of producing neutrons for transmutation applications is with a fission reactor. Although this technology is well established, it is politically and environmentally controversial. Thus, the accelerator demonstration must be low risk, and use established technology to be competitive. Both higher power generators and more efficient amplifiers are desirable options for a second-generation accelerator, but the first design should be conservative.

The power supplies for large accelerators are usually sized 
for only one or two klystror.s, but the existing proton accelerator at the Los Alamos Meson Physics Facility has 44 klystrons in groups of 6 or 7 attached to each power supply. Because klystrons have high levels of isolation between the dc and RF portions of the device, there is no reason why many klystrons cannot be connected to the same power supply. In the design-optimization process, three options were considered: 1,8 , or 188 klystrons connected to each power supply. The power-supply costs per watt become smaller and smaller as the power-supply size is increased, and vendors' estimates were that the cost per watt scales as $P^{-0.5}$, where $P$ is the power rating of the power supply. By doing a simple analysis of the MTBFs for the system, we found that both the 8 and 188 amplifiers connected to a single power supply would meet the $93 \%$ availability goal for the RF system. For the baseline design, we chose the larger power supplies. A complication quickly arose, however.

With the smaller power supplies, whenever one klystron arcs, the power supply is "crowbared" (shorted out by a high speed switch) in a few $\mu$ s, and then the supervisory computer turns the power supply back on once the klystron arc has cleared and the vacuum in the tube is back to nor.nal levels. The large power supply still requires a crowbar for each bank of 8 klystrons, but in addition it needs a fastopening electronic switch in series with each bank of klystrons. The opening switch opens and the crowbar closes whenever a klystron arcs, and the large power supply remains in operation. The series opening switch is a difficult and perhaps expensive technology, and the additional costs may offset the savings from the large power supply. The dynamics of this trade-off are still under investigation.
A major advantage of the large power supply is that it can be built just like half of the large ac-to-dc-to-ac converter systems that are used to join utility grids. These converters can operate with the superlative reliability and efficiency required for utility operation, and they are produced by a few large vendors. The cost per watt is much lower than for a conventional few-megawatt power supply. The AYTY ATW system must operate with an availability of $75 \%$ averaged over each year. Preliminary analyses of the MTBFs of the major subsystems of the RF system (power supplies, power conditioning, the RF amplifier, the cooling system, and the low-level control electronics) indicate that the RF system availability can be in the $95 \%$ range without component redundancy provided that between 50 and $75 \%$ of the RF system failures can be predicted rather than just experienced.

To predict failures, we propose that there be a maintenance shift of 8 hours each week and that the RF system modules be heavily instrumented. Before the maintenance shift, diagnostic readings of the failing components would be printed to guide the maintenance-shift activities. All subsystems not being worked on would remain operational to minimize the start-up transients on the system. The extra diagnostics in the klystron's case include the are rate, gain, and modulation-anode current. These would be monitored and available as charts. Therefore, if a particular klystron would be found either too close to its end-oflife values or if it is trending too rapidly to these values, the klystron would be replaced at the next maintenance shift. In addition, all of the solid-state switches in the power system would be under-rated, instrumented, and designed to fail in the shorted mode. Thus, these components would

\begin{tabular}{|l|c|c|c|r|}
\hline Cavity Type & Frequency & $\begin{array}{l}\text { Number of } \\
\text { RF Genera- } \\
\text { tors }\end{array}$ & $\begin{array}{l}\text { Total RF Pow- } \\
\text { er Required }\end{array}$ & RF Capability \\
\hline RFQ & $350 \mathrm{MHz}$ & 6 & $3.6 \mathrm{MW}$ & $4 \mathrm{MW}$ \\
DTL & $350 \mathrm{MHz}$ & 4 & $4.9 \mathrm{MW}$ & $6 \mathrm{MW}$ \\
BCDTL & $700 \mathrm{MHz}$ & 24 & $22.5 \mathrm{MW}$ & $30 \mathrm{MW}$ \\
CCL & $700 \mathrm{MHz}$ & 342 & $223.2 \mathrm{MW}$ & $342 \mathrm{MW}$ \\
\hline
\end{tabular}

Table 1. Frequency and number of RF amplifiers for the APT/ATW accelerator. 
experience gradual failure and operate well with some failed components. When the instrumentation indicates that the reserve margin is becoming too small, maintenance could be performed at the next opportunity. With strategies such as these, most of the failures could be anticipated with only a small penalty in replacement costs.

\section{Technology Improvements}

The conventional klystron at the 1-MW power level is a well proven but very conservative solution to the amplifier problem for this power system. The costs of a power system generally scale as $P^{-0.5}$, where $P$ here is the RF amplifier unit pawer. Thus, if a $2 \mathrm{MW}$ klystron could be developed, the capital costs of the RF system would be reduced by about $40 \%$, but there would likely be a reliability penalty. The reliability penalty is difficult to estimate, so this trade can only be performed by actually developing new devices and measuring reliability. For a project of this magnitude, alternative types of RF amplifiers should also be developed, because the power savings potential is so large. Two devices that operate much better than the conventional klystron away from saturation are the Klystrode and the multiple-stage depressed-collector klystron $(4,5)$. Neither device has been built at the megawatt power level, but both devices are popular and cost effective in the UHF transmittcr market. Depressed collectors will be very difficult to apply to the high-power klystron, since the basic efficiency and the spent-beam velocity spread are so high. The depressed collector may well be better applies to the Klystrode, where the spend-beam velocity spread is smaller. Some almost undeveloped RF devices have also been proposed for this application, such as the magnicon, a deflection-modulated amplifier, the regotron, a multipleoutput, rebunching type of klystron, and the multiplebeam klystron $(6,7,8)$. The multiple-beam klystron is attractive because a one-megawatt device will operate at about $40 \mathrm{kV}$, compared to the 90 to $100 \mathrm{kV}$ for a high-efficiency conventional klystron. Certainly there will be system reliability advantages for operating at relatively low voltage, and the benefits will occur in reliability and cost. There is a general trend towards higher efficiency as the voltage is increased for any type of tube amplifier, because the effects of space charge are reduced. However, high voltage is not easy to deal with, and low currents and high voltages may be more costly than lower efficiency at a more moderate voltage. Here again, system studies and experimental verification are required to optimize the choices. At the Los Alamos Meson Physics Facility, three separate types of RF generators were developed for the $805 \mathrm{MHz}$ portion of that accelerator, and there were only $44 \mathrm{RF}$ generators needed for that project. With several hundred generators required for the APT/ATW project, several types of generators should be investigated, the the one with the best combination of reliability and capital costs chosen. Only time and a well-funded developmental program will determine the merits of these devices for such applications.

\section{References}

(1) M. Lynch et al.,"Linac design study for an intense neutron-source driver," presented at the 1993 Particle Accelerator Conference, Wishington, May 17-20, 1993.

(2) M. Onoet al., "TRISTAN RF system with normal conducting cavity," KEK Internal Report 87-6, National Laboratory for High linergy Physics, 'Isukuba, Japan, Dec 1987.

(3) H. Frischholz,"The LEP II RF system"paper Sb52, presenled at the 1993 Particle Accelerator Conference, Washington, May 17-211, 1993.

(4) M.B.Schrader and D.H. Priest, "PF sources for particle acceleratorsa progress report", Proceedings of the 1981 Linear Acceleratur Conference, Los Alamos report LA-9234-C, p. 326, Santa Ve, (ktober I'y81. (5) E.W. McCune, "Klystron performance using a multistage depressed collector", presented at the 1987 IEEE Electron Device Cunference. pp. 157-159, Dec. 1987.

(6) M.M. Karliner et al.,"The Magnicon-an advanced version of the gyrocon." Nuclear Inst. and Methods in Physics Research Vol $\mathrm{A} 26 \%$. pl. 459-473, 1988.

(7) B.P. Murin, A.P. Durkin, O.Yu. Shlygin, I.V. Shumakov, "Superhigh -power RF regotron-type generator for linear accelerator with high be:am current," Proceedings of the 1990 Linear Accelerator Conference. Albu querque, Sept 10-14, 1990 (Los Alamos Report 1A-12004-(')

(8) C. Bearzatto, M. Bres, G. Faillon, "Advantages of multiple beam klystrons," presented at the 1992 Fusion Energy Conference, (iarmisch. May 4-5, 1992. 

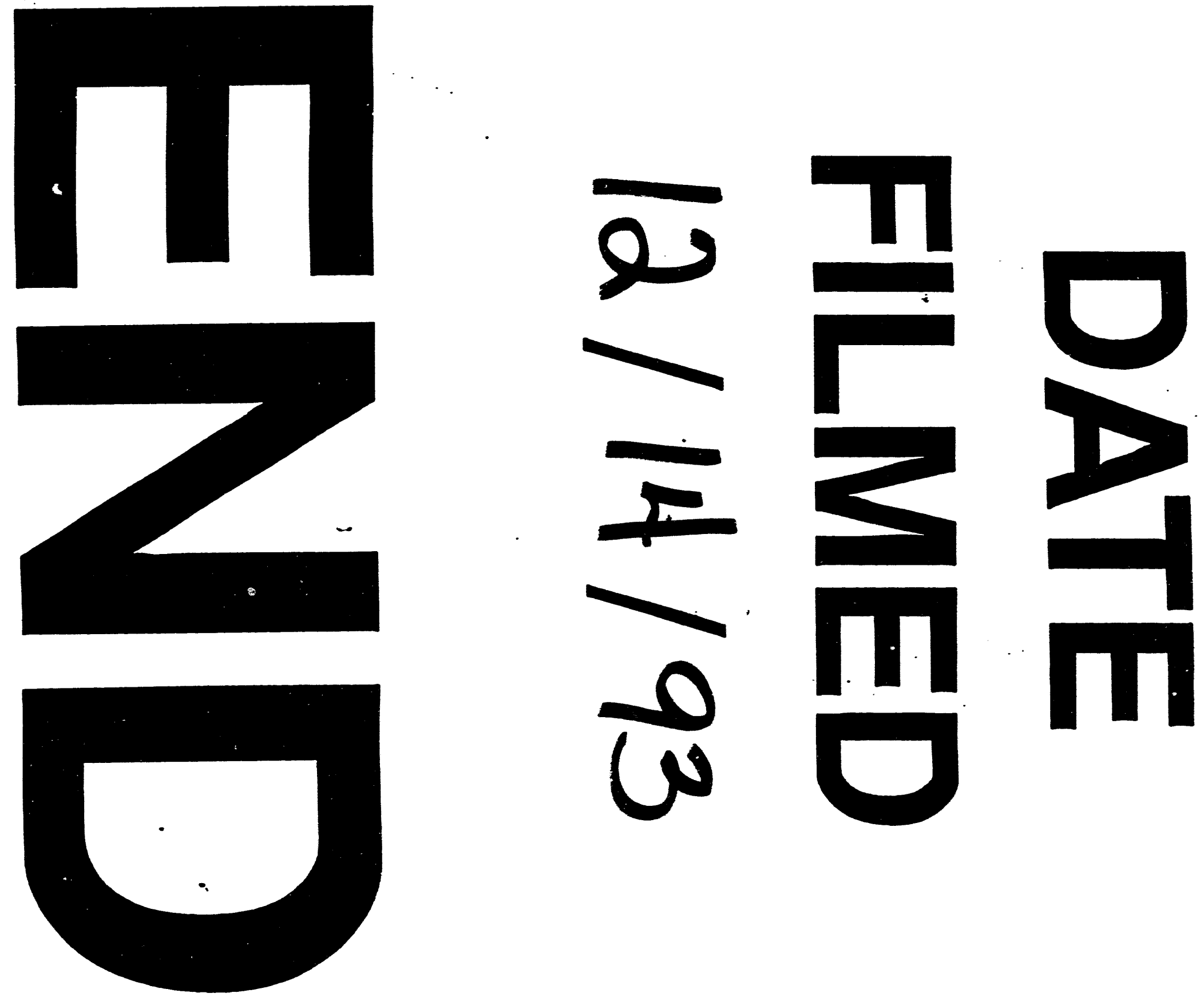
